\title{
Glomerular Filtration Response to Acute Loading with Protein from Different Sources in Healthy Volunteers and Diabetic Patients
}

\author{
Hiroshi Nakamura, Masatoshi Yamazaki, Yasuko \\ Chiba, Noriko Tamura, Takeshi Momotsu, Seiki Ito, \\ Akira Shibata, Kyuzi Kamor* and Tohru Yamaji $\dagger$ \\ The First Department of Internal Medicine, Niigata \\ University School of Medicine, Niigata, 951, *Nagaoka Red \\ Cross Hospital, Niigata, 940 and †the Third Department of \\ Internal Medicine, Faculty of Medicine, University of \\ Tokyo, Tokyo, 113
}

Nakamura, H., Yamazaki, M., Chiba, Y., Tamura, N., Momotsu, T., Ito, S., Shibata, A., Kamoi, K. and Yamaj, T. Glomerular Filtration Response to Acute Loading with Protein from Different Sources in Healthy Volunteers and Diabetic Patients. Tohoku J. Exp. Med., 1990, $162(3), 269-278 —$ To evaluate the effects of acute protein loading on the glomerular filtration rate, albumin excretion rate and concentration of plasma amino acids, ten healthy volunteers and six type 2 diabetic patients with normoalbuminuria were studied before and after eating 0.7 $\mathrm{g} / \mathrm{kg}$ body weight of tuna fish, boiled egg white, cheese or tofu (bean curd) on separate days. Furthermore, to study the possible role of glucagon, growth hormone, atrial natriuretic peptide and kallikrein in the responses of glomerular filtration rate to protein, these substances were measured before and after ingestion of tuna fish or egg white in six healthy volunteers. In healthy subjects, glomerular filtration rate increased significantly $(p<0.01)$ from $98.1 \pm 4.2 \mathrm{ml} / \mathrm{min}$ during the baseline period to $129.9 \pm 6.6 \mathrm{ml} / \mathrm{min}$ after ingestion of tuna fish. No significant differences were seen between glomerular filtration rate before and after ingestion of egg white, cheese or bean curd. No significant differences were observed between the baseline albumin excretion rate and that after loading with any of the four kinds of protein. Plasma concentrations of alanine, glycine and arginine (amino acids known to induce glomerular hyperfiltration) increased to a greater degree after ingestion of tuna fish than after administration of the other meals. Diabetic subjects and healthy volunteers had similar responses. Plasma glucagon and growth hormone concentrations increased after ingestion of the tuna fish meal or egg white. Plasma atrial natriuretic peptide concentration and urinary kallikrein excretion were unaffected by ingestion of these two kinds of protein. These findings suggest that responses of glomerular filtration rate to acute protein loading may differ depending on the protein ingested, and that these reponses may not be directly induced by glucagon, growth hormone, atrial natriuretic peptide or kallikrein. —— - diabetic nephropathy; acute protein loading; hyperfiltration ; composition of amino acids 
The presence of an elevated glomerular filtration rate (GFR) in uncomplicated type 1 (insulin-dependent) diabetic patients has been known for many years (Mogensen 1971, 1976). It was reported that glomerular hyperfiltration in type 1 diabetes with early stage diabetic nephropathy might be important in the subsequent development of late diabetic nephropathy (Mogensen 1971, 1976). Although the exact mechanism inducing glomerular hyperfiltration remains to be elucidated, many factors, such as glucose (Greene et al. 1987), ketone bodies (Trevisan et al. 1987), dietary protein (Bosch et al. 1983, 1984, 1986 ; Jones et al. 1985; Hostetter 1986; Dhaene et al. 1987) and prostaglandins (Krishna et al. 1988), have been implicated.

It was recently reported that a high-protein diet produces a significant increase in GFR (Bosch et al. 1983, 1984, 1986 ; Jones et al. 1985 ; Hostetter 1986 ; Dhaene et al. 1987) and that low-protein alimentation might prevent the progression of renal failure in patients with diabetic nephropathy (Gingliano et al. 1986 ; Evanoff et al. 1987 ; Wiseman et al. 1987). However, low-protein alimentation should be approached with caution, because hypoproteinemia was often observed in protein-restricted rats with renal failure (Motomura et al. 1988). To date, mainly animal proteins have been used to study the effects of acute protein loading on renal function. It was reported that GFR increased after ingestion of animal proteins (Bosch et al. 1983, 1984, 1986 ; Hostetter 1986). These findings, together with the finding that low-protein alimentation may have the abovementioned harmful side effects, led us to investigate whether diabetic nephropath$\mathrm{y}$ is influenced by the quality as well as the quantity of dietary protein. In a previous report, we examined the influence of proteins from two different sources on renal function in healthy volunteers and diabetic subjects (Nakamura et al. 1989). Our study showed that ingestion of cooked soybean protein does not increase GFR. This result raised the possibility that not all animal proteins might affect the GFR. Therefore, we studied the effects of acute protein loading of four different protein sources on renal function in healthy volunteers and diabetic patients.

The exact mechanism of protein-induced glomerular hyperfiltration remains to be elucidated. It has, however, been reported that glucagon (Parving et al. 1977), growth hormone (Corvilain and Abramow 1962), atrial natriuretic peptide (ANP) (Fried and McCoy 1986) and kallikrein-kinin system (Levy et al. 1977) induced glomerular hyperfiltration. Another aim of this study, therefore, was to examine the role of these substances in mediating glomerular hyperfiltration.

\section{Subjects and Methods}

The subjects comprised ten healthy volunteers (6 men and 4 women, aged $49.1 \pm 8.9$ years, BMI $22.0 \pm 1.9 \mathrm{~kg} / \mathrm{m}^{2}$ ) and six type 2 diabetic patients (4 men and 2 women, aged $48.7 \pm 10.0$ years, BMI $21.9 \pm 1.8 \mathrm{~kg} / \mathrm{m}^{2}$ ). All were normotensive and had normal renal function as evidenced by normal serum creatinine concentration and urinalysis. Three 
diabetic patients were given oral hypoglycemic agent, and the others were treated with diet therapy. All diabetes were in resonable metabolic control (fasting blood glucose $112.0 \pm 5.8$ $\mathrm{mg} / 100 \mathrm{ml}, \mathrm{HbAlc} 5.9 \pm 0.2 \%$, normal range $4.6-5.7 \%$ ). Duration of diabetes was $4.4 \pm 1.0$ years. Protein loading tests were performed in the fasting state. Subjects began the test by receiving oral hydration with $400 \mathrm{ml}$ of water. Baseline and test GFRs were calculated from endogenous creatinine clearance. After the baseline creatinine clearances were measured, the subjects ingested on separate days $0.7 \mathrm{~g} / \mathrm{kg}$ body weight of protein in the form of cooked tuna fish, boiled, salted egg white, cheese or tofu (bean curd) with soy sauce. The composition of each test meal is shown in Table 1. After protein loading, the subjects ingested $100 \mathrm{ml}$ of water each hour. Urine volume was recorded, and creatinine and albumin concentrations in both urine and plasma were measured hourly and the albumin excretion rates (AER) were calculated. Plasma amino acid concentrations were also measured before and after protein loading.

To study the effect of protein loading on the plasma concentrations of glucagon, growth hormone and ANP and on urinary kallikrein excretion, six additional healthy male subjects aged 23 to 28 years received acute loading tests of two kinds of protein (tuna fish and egg white). Plasma concentrations of glucagon, growth hormone and ANP were measured hourly. Urinary concentrations of kallikrein were also measured hourly, and kallikrein excretion rates were calculated.

Measurements of albumin in urine were carried out by double-antibody radioimmunoas-

TABLE 1. Composition of the four test meals

\begin{tabular}{lcccc}
\hline & Tuna & Egg & Cheese & Tofu \\
\hline Carbohydrates (g/g protein) & 0.14 & 0.09 & 0.09 & 0.12 \\
Salt (g/g protein) & 0.11 & 0.10 & 0.06 & 0.10 \\
Water (g/g protein) & 2.96 & 8.43 & 2.76 & 3.47 \\
Phosphate (mg/g protein) & 15.4 & 1.10 & 31.7 & 12.5 \\
Amino acid (mg/g protein) & & & & \\
Aspartic acid & 92 & 110 & 76 & 120 \\
Threonine & 43 & 45 & 35 & 41 \\
Serine & 44 & 64 & 50 & 51 \\
Glutamic acid & 140 & 140 & 210 & 190 \\
Proline & 32 & 36 & 110 & 59 \\
Glycine & 54 & 37 & 18 & 46 \\
Alanine & 66 & 62 & 29 & 47 \\
Valine & 48 & 73 & 68 & 56 \\
Cystine & 10 & 31 & 8 & 18 \\
Methionine & 29 & 39 & 27 & 15 \\
Isoleucine & 48 & 56 & 53 & 54 \\
Leucine & 80 & 89 & 98 & 89 \\
Tyrosine & 37 & 40 & 55 & 43 \\
Phenylalanine & 40 & 60 & 53 & 33 \\
Histidine & 93 & 25 & 31 & 29 \\
Tryptophan & 13 & 16 & 13 & 15 \\
Lysine & 87 & 69 & 82 & 68 \\
Arginine & 66 & 59 & 34 & 81 \\
\hline
\end{tabular}


say according to a method previously reported (Ito et al. 1989). Serum albumin levels were measured by laser nephelometry. Creatinine concentrations in urine and serum were measured by Folin's method. Plasma amino acid concentrations were measured by an auto-analyzer (System 6300 : Beckman, Tokyo). Plasma glucagon levels were measured by double-antibody radioimmunoassay using specific antisera against pancreatic glucagon (Sugimoto 1981). Plasma growth hormone and ANP (Yamaji et al. 1985) were measured by radioimmunoassay, as was urinary kallikrein (PEG method) (Kato et al. 1980).

The GFR was always corrected for body surface area and was expressed per $1.73 \mathrm{~m}^{2}$. Body surface area was calculated from height and weight. All data are expressed as mean values \pm S.D.. The significance between the means was calculated using Student's $t$-test or Welch's test.

\section{Results}

\section{Renal response to protein loading}

The results of the protein loading tests are shown in Tables 2 and 3 . In healthy volunteers, GFR increased significantly $(p<0.01)$ after ingestion of tuna fish : from $98.1 \pm 4.2 \mathrm{ml} / \mathrm{min} / 1.73 \mathrm{~m}^{2}$ during the baseline period to $129.9 \pm 6.6 \mathrm{ml} /$ $\min / 1.73 \mathrm{~m}^{2}$. In diabetic patients, GFR also increased significantly after tuna fish ingestion $\left(96.7 \pm 6.2\right.$ to $\left.130.8 \pm 7.5 \mathrm{ml} / \mathrm{min} / 1.73 \mathrm{~m}^{2}\right)$. No significant differences were found between GFR before and after ingestion of egg white, cheese, or bean curd (Fig. 1). In all subjects, plasma concentration of serum creatinine increased significantly after ingestion of tuna fish : from $0.88 \pm 0.09 \mathrm{mg}$ / $100 \mathrm{ml}$ to $1.42 \pm 0.10 \mathrm{mg} / 100 \mathrm{ml}$. No significant differences in plasma creatinine concentrations were shown before and after ingestion of the other three meals. No significant differences in AER were observed before and after loading with any of the four kinds of protein. In all subjects, mean atrial blood pressure and blood glucose levels did not change during study.

TABLE 2. Changes in GFR ( $\left.\mathrm{ml} / \mathrm{min} / 1.73 \mathrm{~m}^{2}\right)$ following protein loading

\begin{tabular}{|c|c|c|c|c|c|c|c|c|}
\hline \multirow{3}{*}{ Meal } & \multicolumn{4}{|c|}{ Healthy subjects } & \multicolumn{4}{|c|}{ Diabetic subjects } \\
\hline & \multirow{2}{*}{ Pre-meal } & \multicolumn{3}{|c|}{ Post-meal } & \multirow{2}{*}{ Pre-meal } & \multicolumn{3}{|c|}{ Post-meal } \\
\hline & & $1 \mathrm{hr}$ & $2 \mathrm{hr}$ & $3 \mathrm{hr}$ & & $1 \mathrm{hr}$ & $2 \mathrm{hr}$ & $3 \mathrm{hr}$ \\
\hline Tuna & $\begin{array}{r}98.1 \\
\pm 4.2\end{array}$ & $\begin{array}{l}110.6 \\
\pm 5.9\end{array}$ & $\begin{array}{l}120.7^{*} \\
\pm 7.7\end{array}$ & $\begin{array}{l}129.9^{*} \\
\pm 6.6\end{array}$ & $\begin{array}{r}96.7 \\
\pm 6.2\end{array}$ & $\begin{array}{l}109.1 \\
\pm 7.3\end{array}$ & $\begin{array}{l}121.8^{*} \\
\pm 8.1\end{array}$ & $\begin{array}{l}130.8^{*} \\
\pm 7.5\end{array}$ \\
\hline Egg & $\begin{array}{r}99.0 \\
\pm 4.6\end{array}$ & $\begin{array}{r}97.6 \\
\pm 3.8\end{array}$ & $\begin{array}{l}100.0 \\
\pm 5.7\end{array}$ & $\begin{array}{r}99.5 \\
\pm 4.7\end{array}$ & $\begin{array}{r}97.1 \\
\pm 5.2\end{array}$ & $\begin{array}{r}98.7 \\
\pm 4.6\end{array}$ & $\begin{array}{r}97.2 \\
\pm 3.6\end{array}$ & $\begin{array}{l}101.0 \\
\pm 4.2\end{array}$ \\
\hline Cheese & $\begin{array}{r}98.9 \\
\pm 5.4\end{array}$ & $\begin{array}{r}97.9 \\
\pm 2.9\end{array}$ & $\begin{array}{r}98.2 \\
\pm 3.3\end{array}$ & $\begin{array}{r}98.7 \\
\pm 2.8\end{array}$ & $\begin{array}{r}99.9 \\
\pm 6.1\end{array}$ & $\begin{array}{r}97.9 \\
\pm 7.6\end{array}$ & $\begin{array}{r}98.1 \\
\pm 5.1\end{array}$ & $\begin{array}{r}92.1 \\
\pm 4.8\end{array}$ \\
\hline Tofu & $\begin{array}{r}98.9 \\
\pm 4.5\end{array}$ & $\begin{array}{r}94.7 \\
\pm 5.6\end{array}$ & $\begin{array}{r}99.7 \\
\pm 4.0\end{array}$ & $\begin{array}{r}97.3 \\
\pm 4.0\end{array}$ & $\begin{array}{r}97.5 \\
\pm 4.2\end{array}$ & $\begin{array}{r}97.5 \\
\pm 5.7\end{array}$ & $\begin{array}{r}98.4 \\
\pm 3.9\end{array}$ & $\begin{array}{r}96.2 \\
\pm 4.6\end{array}$ \\
\hline
\end{tabular}

Values are means \pm s.D..

${ }^{*} p<0.01$ vs. baseline values. 
TABLE 3. Changes in $A E R$ ( $\mu \mathrm{g} / \mathrm{min})$ following protein loading

\begin{tabular}{|c|c|c|c|c|c|c|c|c|}
\hline \multirow{3}{*}{ Meal } & \multicolumn{4}{|c|}{ Healthy subjects } & \multicolumn{4}{|c|}{ Diabetic subjects } \\
\hline & \multirow{2}{*}{ Pre-meal } & \multicolumn{3}{|c|}{ Post-meal } & \multirow{2}{*}{ Pre-meal } & \multicolumn{3}{|c|}{ Post-meal } \\
\hline & & $1 \mathrm{hr}$ & $2 \mathrm{hr}$ & $3 \mathrm{hr}$ & & $1 \mathrm{hr}$ & $2 \mathrm{hr}$ & $3 \mathrm{hr}$ \\
\hline Tuna & $\begin{array}{r}7.9 \\
\pm 5.7\end{array}$ & $\begin{array}{r}9.9 \\
\pm 7.7\end{array}$ & $\begin{array}{r}6.4 \\
\pm 5.0\end{array}$ & $\begin{array}{r}6.2 \\
\pm 4.5\end{array}$ & $\begin{array}{r}7.9 \\
\pm 4.5\end{array}$ & $\begin{array}{r}8.9 \\
\pm 4.1\end{array}$ & $\begin{array}{r}7.4 \\
\pm 3.9\end{array}$ & $\begin{array}{r}6.5 \\
\pm 3.7\end{array}$ \\
\hline Egg & $\begin{array}{r}7.1 \\
+5.9\end{array}$ & $\begin{array}{r}4.9 \\
\pm 3.8\end{array}$ & $\begin{array}{r}5.3 \\
\pm 4.0\end{array}$ & $\begin{array}{r}6.6 \\
\pm 4.2\end{array}$ & $\begin{array}{r}6.9 \\
\pm 4.1\end{array}$ & $\begin{array}{r}5.0 \\
\pm 3.4\end{array}$ & $\begin{array}{r}7.3 \\
+4.8\end{array}$ & $\begin{array}{r}6.6 \\
\pm 4.7\end{array}$ \\
\hline Cheese & $\begin{array}{r}6.2 \\
\pm 3.4\end{array}$ & $\begin{array}{r}7.2 \\
\pm 5.1\end{array}$ & $\begin{array}{r}5.9 \\
+4.6\end{array}$ & $\begin{array}{r}7.4 \\
\pm 5.7\end{array}$ & $\begin{array}{r}7.2 \\
\pm 3.8\end{array}$ & $\begin{array}{r}6.9 \\
\pm 3.9\end{array}$ & $\begin{array}{r}5.2 \\
\pm 2.9\end{array}$ & $\begin{array}{r}6.4 \\
\pm 5.0\end{array}$ \\
\hline Tofu & $\begin{array}{r}6.7 \\
\pm 4.6\end{array}$ & $\begin{array}{r}8.4 \\
+6.3\end{array}$ & $\begin{array}{r}7.4 \\
\pm 6.2\end{array}$ & $\begin{array}{r}8.6 \\
+6.4\end{array}$ & $\begin{array}{r}6.8 \\
\pm 5.3\end{array}$ & $\begin{array}{r}7.1 \\
\pm 4.6\end{array}$ & $\begin{array}{r}8.1 \\
\pm 5.9\end{array}$ & $\begin{array}{r}8.6 \\
+5.7\end{array}$ \\
\hline
\end{tabular}

Values are means \pm s.D..

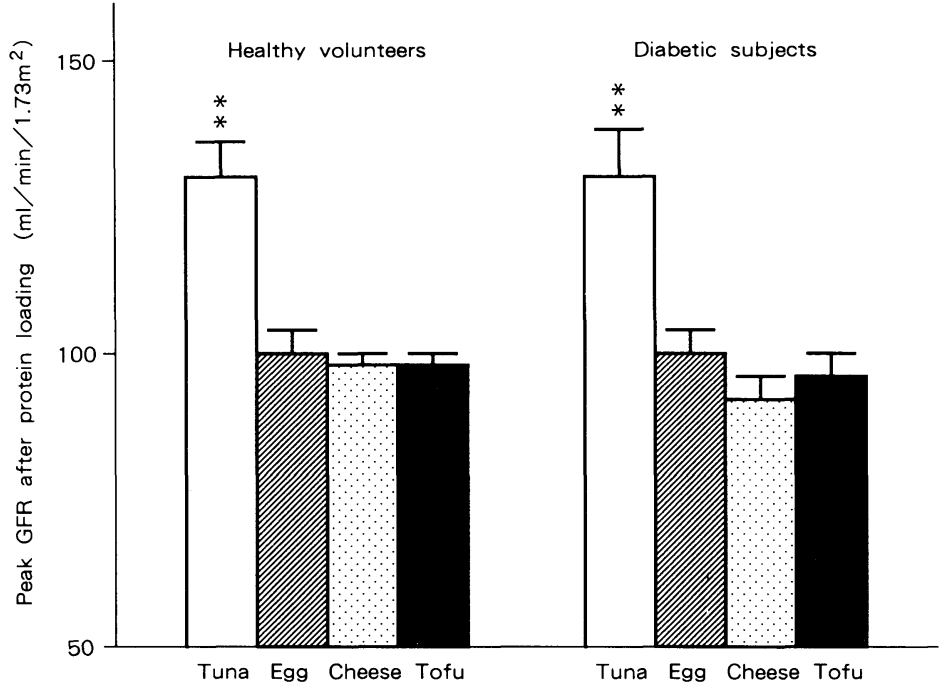

Fig. 1. Chages in GFR following protein loads. GFR increased significantly after ingestion of tuna fish meal ( ${ }^{* *} p<0.01$ vs. baseline values).

\section{Changes in plasma amino acids following protein loading}

The results of the plasma amino acid analysis are shown in Table 4. Almost all amino acids increased in plasma after ingestion of each of the four kinds of protein. Ingestion of tuna fish caused significantly greater increases in plasma concentration of total amino acids than the other meals. Plasma concentrations of alanine, glycine and arginine, amino acids known to induce glomerular hyperfiltration, increased more after the ingestion of tuna fish than after the other meals. 
TABLE 4. Increases in plasma amino acid concentrations following protein loads

\begin{tabular}{|c|c|c|c|c|c|c|c|c|}
\hline & \multicolumn{4}{|c|}{ Healthy subjects } & \multicolumn{4}{|c|}{ Diabetic subjects } \\
\hline & Tuna & Egg & Cheese & Tofu & Tuna & Egg & Cheese & Tofu \\
\hline Total & $\begin{array}{r}2065 \\
\pm 224\end{array}$ & $\begin{array}{l}1191^{* *} \\
\pm 168\end{array}$ & $\begin{array}{l}1182^{* *} \\
\pm 119\end{array}$ & $\begin{array}{l}1079^{* *} \\
\pm 153\end{array}$ & $\begin{array}{r}1998 \\
\pm 189\end{array}$ & $\begin{array}{l}1062^{* *} \\
\pm 167\end{array}$ & $\begin{array}{c}1059^{* *} \\
\pm 148\end{array}$ & $\begin{array}{c}1176^{* *} \\
\pm 196\end{array}$ \\
\hline Aspartic acid & $\begin{array}{r}4.5 \\
\pm 1.8\end{array}$ & $\begin{array}{l}0.0^{* *} \\
\pm 0.5\end{array}$ & $\begin{array}{l}1.8^{* *} \\
\pm 0.3\end{array}$ & $\begin{array}{r}2.2^{*} \\
\pm 1.4\end{array}$ & $\begin{array}{r}2.5 \\
\pm 0.4\end{array}$ & $\begin{array}{l}0.0^{* *} \\
\pm 0.3\end{array}$ & $\begin{array}{l}0.4^{* *} \\
\pm 0.5\end{array}$ & $\begin{array}{ll} & 1.3^{* *} \\
\pm & 0.3\end{array}$ \\
\hline Threonine & $\begin{array}{r}127.6 \\
\pm 49.8\end{array}$ & $\begin{array}{l}18.4^{* *} \\
\pm 8.8\end{array}$ & $\begin{array}{l}20.9^{* *} \\
\pm 7.1\end{array}$ & $\begin{array}{l}38.5^{* *} \\
\pm 9.7\end{array}$ & $\begin{array}{r}46.5 \\
\pm 9.0\end{array}$ & $\begin{array}{l}0.0^{* *} \\
\pm 2.7\end{array}$ & $\begin{array}{l}12.9^{* *} \\
\pm 5.1\end{array}$ & $\begin{array}{l}28.5^{* *} \\
\pm 7.2\end{array}$ \\
\hline Serine & $\begin{array}{r}61.8 \\
+12.4\end{array}$ & $\begin{array}{r}69.1 \\
\pm 11.7\end{array}$ & $\begin{array}{r}56.2 \\
+13.6\end{array}$ & $\begin{array}{r}52.7 \\
\pm 14.2\end{array}$ & $\begin{array}{r}32.8 \\
+12.8\end{array}$ & $\begin{array}{r}35.4 \\
+14.6\end{array}$ & $\begin{array}{r}27.1 \\
\pm 15.1\end{array}$ & $\begin{array}{r}27.5 \\
\pm 12.0\end{array}$ \\
\hline Glutamic acid & $\begin{array}{r}30.6 \\
\pm 9.3\end{array}$ & $\begin{array}{l}15.3^{* *} \\
\pm 7.3\end{array}$ & $\begin{array}{l}13.2^{* *} \\
\pm 8.1\end{array}$ & $\begin{array}{r}13.9 \\
\pm 6.5\end{array}$ & $\begin{array}{r}28.1 \\
\pm 7.9\end{array}$ & $\begin{array}{l}9.1^{* *} \\
\pm 5.2\end{array}$ & $\begin{array}{l}8.8^{* *} \\
\pm 3.4\end{array}$ & $\begin{array}{c}17.3^{*} \\
\pm 6.0\end{array}$ \\
\hline Proline & $\begin{array}{r}99.9 \\
\pm 33.1\end{array}$ & $\begin{array}{r}121.7 \\
\pm 32.6\end{array}$ & $\begin{array}{r}102.4 \\
\pm 21.8\end{array}$ & $\begin{array}{r}81.8 \\
+21.2\end{array}$ & $\begin{array}{r}63.1 \\
+20.8\end{array}$ & $\begin{array}{r}87.2 \\
\pm 29.9\end{array}$ & $\begin{array}{r}69.0 \\
\pm 21.5\end{array}$ & $\begin{array}{r}56.9 \\
+19.8\end{array}$ \\
\hline Glycine & $\begin{array}{r}75.0 \\
+17.8\end{array}$ & $\begin{array}{l}10.7^{* *} \\
\pm 9.8\end{array}$ & $\begin{array}{l}25.5^{* *} \\
\pm 8.0\end{array}$ & $\begin{aligned} & 11.3^{* *} \\
\pm & 10.8\end{aligned}$ & $\begin{array}{r}68.0 \\
\pm 9.4\end{array}$ & $\begin{array}{l}5.2^{* *} \\
\pm 3.6\end{array}$ & $\begin{array}{l}7.9^{* *} \\
\pm 3.4\end{array}$ & $\begin{array}{l}0.0^{* *} \\
\pm 0.4\end{array}$ \\
\hline Alanine & $\begin{array}{r}167.3 \\
\pm 35.2\end{array}$ & $\begin{aligned} & 39.4^{* * *} \\
\pm & 15.5\end{aligned}$ & $\begin{aligned} & 42.5^{* *} \\
\pm & 14.9\end{aligned}$ & $\begin{aligned} & 15.1^{* *} \\
\pm & 12.3\end{aligned}$ & $\begin{array}{r}187.6 \\
\pm 21.0\end{array}$ & $\begin{aligned} & 41.8^{* *} \\
\pm & 16.7\end{aligned}$ & $\begin{aligned} & 35.4^{* *} \\
\pm & 13.8\end{aligned}$ & $\begin{aligned} & 10.3^{* *} \\
\pm & 10.6\end{aligned}$ \\
\hline Valine & $\begin{array}{r}305.3 \\
\pm 51.2\end{array}$ & $\begin{array}{l}111.7^{* *} \\
\pm 46.1\end{array}$ & $\begin{array}{l}120.2^{* *} \\
\pm 49.0\end{array}$ & $\begin{array}{l}136.5^{* *} \\
\pm 38.9\end{array}$ & $\begin{array}{r}289.9 \\
\pm 49.2\end{array}$ & $\begin{array}{l}142.0^{* *} \\
\pm 40.0\end{array}$ & $\begin{array}{l}133.9^{* *} \\
\pm 40.6\end{array}$ & $\begin{array}{l}161.2^{* *} \\
\pm 38.4\end{array}$ \\
\hline Cystine & $\begin{array}{r}6.1 \\
+1.5\end{array}$ & $\begin{aligned} & 1.2^{* *} \\
\pm & 1.0\end{aligned}$ & $\begin{array}{c}2.1^{* *} \\
\pm 1.2\end{array}$ & $\begin{array}{c}0.0^{* *} \\
\pm 0.9\end{array}$ & $\begin{array}{r}7.4 \\
+1.5\end{array}$ & $\begin{aligned} & 1.0^{* *} \\
\pm & 1.3\end{aligned}$ & $\begin{array}{l}1.6^{* *} \\
\pm 1.0\end{array}$ & $\begin{aligned} & 0.0^{* *} \\
\pm & 0.8\end{aligned}$ \\
\hline Methionine & $\begin{array}{r}64.8 \\
\pm 8.0\end{array}$ & $\begin{array}{l}20.6^{* *} \\
\pm 5.1\end{array}$ & $\begin{array}{l}18.8^{* *} \\
\pm 5.6\end{array}$ & $\begin{array}{l}2.9^{* *} \\
\pm 2.4\end{array}$ & $\begin{array}{r}78.1 \\
\pm 6.4\end{array}$ & $\begin{array}{l}10.7^{* *} \\
\pm 3.5\end{array}$ & $\begin{array}{l}13.4^{* *} \\
\pm 2.7\end{array}$ & $\begin{aligned} & 2.2^{* *} \\
\pm & 1.9\end{aligned}$ \\
\hline Isoleucine & $\begin{array}{r}149.7 \\
\pm 12.6\end{array}$ & $\begin{array}{r}58.3^{* *} \\
\pm 11.2\end{array}$ & $\begin{aligned} & 44.6^{* *} \\
\pm & 12.8\end{aligned}$ & $\begin{aligned} & 64.2^{* *} \\
\pm & 10.3\end{aligned}$ & $\begin{array}{r}109.7 \\
\pm 17.4\end{array}$ & $\begin{aligned} & 49.2^{* *} \\
\pm & 12.3\end{aligned}$ & $\begin{aligned} & 48.9^{* *} \\
\pm & 16.9\end{aligned}$ & $\begin{array}{r}87.0^{*} \\
\pm 12.2\end{array}$ \\
\hline Leucine & $\begin{array}{r}235.1 \\
\pm 23.3\end{array}$ & $\begin{aligned} & 91.3^{* *} \\
\pm & 19.0\end{aligned}$ & $\begin{array}{l}105.0^{* *} \\
\pm 18.5\end{array}$ & $\begin{array}{l}102.5^{* *} \\
\pm 13.1\end{array}$ & $\begin{array}{r}246.7 \\
\pm 26.4\end{array}$ & $\begin{array}{l}100.9^{* *} \\
\pm 21.0\end{array}$ & $\begin{aligned} & 99.9^{* *} \\
+ & 17.8\end{aligned}$ & $\begin{array}{l}114.2^{* *} \\
\pm 12.5\end{array}$ \\
\hline Tyrosine & $\begin{array}{r}61.2 \\
\pm 10.6\end{array}$ & $\begin{array}{l}36.4^{* *} \\
\pm 8.5\end{array}$ & $\begin{array}{l}35.4^{* *} \\
\pm 7.2\end{array}$ & $\begin{array}{c}38.3^{* *} \\
\pm 9.1\end{array}$ & $\begin{array}{r}62.4 \\
\pm 12.4\end{array}$ & $\begin{array}{l}32.5^{* *} \\
\pm 8.7\end{array}$ & $\begin{array}{l}41.6^{* *} \\
\pm 6.9\end{array}$ & $\begin{array}{l}30.4^{* *} \\
\pm 8.8\end{array}$ \\
\hline Phenylalanine & $\begin{array}{r}33.9 \\
\pm 6.2\end{array}$ & $\begin{aligned} & 0.0^{* *} \\
\pm & 3.4\end{aligned}$ & $\begin{array}{l}12.6^{* *} \\
\pm 3.5\end{array}$ & $\begin{array}{r}34.2 \\
\pm 4.9\end{array}$ & $\begin{array}{r}35.4 \\
\pm 7.1\end{array}$ & $\begin{array}{l}0.0^{* * *} \\
\pm 3.0\end{array}$ & $\begin{array}{l}11.5^{* *} \\
\pm 6.4\end{array}$ & $\begin{array}{l}21.6^{* *} \\
\pm 5.8\end{array}$ \\
\hline Histidine & $\begin{array}{r}101.0 \\
\pm 13.5\end{array}$ & $\begin{array}{l}0.0^{* *} \\
\pm 5.8\end{array}$ & $\begin{array}{l}0.8^{* *} \\
\pm 6.7\end{array}$ & $\begin{array}{l}11.6^{* *} \\
\pm 9.7\end{array}$ & $\begin{array}{r}97.2 \\
\pm 10.6\end{array}$ & $\begin{aligned} & 0.0^{* * *} \\
\pm & 6.4\end{aligned}$ & $\begin{aligned} & 8.7^{* *} \\
\pm & 9.8\end{aligned}$ & $\begin{aligned} & 5.4^{* *} \\
\pm & 4.9\end{aligned}$ \\
\hline Tryptophan & $\begin{array}{r}35.0 \\
\pm 6.2\end{array}$ & $\begin{array}{l}0.2^{* *} \\
\pm 3.1\end{array}$ & $\begin{array}{l}9.9^{* *} \\
\pm 4.8\end{array}$ & $\begin{array}{l}12.1^{* *} \\
\pm 5.5\end{array}$ & $\begin{array}{r}33.4 \\
\pm 6.8\end{array}$ & $\begin{array}{l}0.0^{* *} \\
\pm 2.1\end{array}$ & $\begin{array}{l}5.6^{* *} \\
\pm 3.4\end{array}$ & $\begin{array}{l}7.3^{* *} \\
\pm 4.7\end{array}$ \\
\hline Lysine & $\begin{array}{r}287.0 \\
\pm 25.3\end{array}$ & $\begin{aligned} & 51.3^{* *} \\
\pm & 19.9\end{aligned}$ & $\begin{aligned} & 68.4^{* *} \\
\pm & 17.9\end{aligned}$ & $\begin{aligned} & 81.9^{* *} \\
\pm & 20.6\end{aligned}$ & $\begin{array}{r}254.3 \\
\pm 28.4\end{array}$ & $\begin{aligned} & 46.9^{* *} \\
\pm & 16.3\end{aligned}$ & $\begin{aligned} & 79.2^{* *} \\
\pm & 19.0\end{aligned}$ & $\begin{aligned} & 81.6^{* *} \\
\pm & 16.8\end{aligned}$ \\
\hline Arginine & $\begin{array}{r}121.7 \\
\pm 17.6\end{array}$ & $\begin{aligned} & 68.6^{* *} \\
\pm & 16.4\end{aligned}$ & $\begin{aligned} & 57.9^{* *} \\
\pm & 15.4\end{aligned}$ & $\begin{aligned} & 65.0^{* *} \\
\pm & 16.8\end{aligned}$ & $\begin{array}{r}108.5 \\
\pm 19.6\end{array}$ & $\begin{aligned} & 49.3^{* *} \\
+ & 15.2\end{aligned}$ & $\begin{aligned} & 50.8^{* *} \\
\pm & 16.8\end{aligned}$ & $\begin{aligned} & 50.4^{* *} \\
\pm & 16.3\end{aligned}$ \\
\hline
\end{tabular}

Values are expressed as mean increases \pm s.D. in amino acid concentration (nmol/ $\mathrm{ml}$ ) above baseline values after protein intake. Different from the respective tuna fish meal intake : ${ }^{*} p<0.05,{ }^{* *} p<0.01$. 
TABLE 5. Changes in glucagon, growth hormone, ANP, and kallikrein following protein loads in normal volunteers

\begin{tabular}{|c|c|c|c|c|c|c|c|c|}
\hline & \multicolumn{4}{|c|}{ Tuna fish } & \multicolumn{4}{|c|}{ Egg white } \\
\hline & \multirow{2}{*}{ Pre-meal } & \multicolumn{3}{|c|}{ Post-meal } & \multirow{2}{*}{ Pre-meal } & \multicolumn{3}{|c|}{ Post-meal } \\
\hline & & $1 \mathrm{hr}$ & $2 \mathrm{hr}$ & $3 \mathrm{hr}$ & & $1 \mathrm{hr}$ & $2 \mathrm{hr}$ & $3 \mathrm{hr}$ \\
\hline $\begin{array}{l}\text { Glucagon } \\
\text { (ng/ml) }\end{array}$ & $\begin{array}{r}81.3 \\
\pm 16.8\end{array}$ & $\begin{array}{c}125.0^{*} \\
\pm 21.4\end{array}$ & $\begin{array}{c}126.7^{*} \\
\pm 20.5\end{array}$ & $\begin{array}{l}140.7^{* *} \dagger \\
\pm 30.6\end{array}$ & $\begin{array}{r}81.0 \\
+18.7\end{array}$ & $\begin{array}{c}108.3^{*} \\
\pm 25.4\end{array}$ & $\begin{array}{c}103.2^{*} \\
\pm 31.0\end{array}$ & $\begin{array}{c}102.8^{*} \\
\pm 25.4\end{array}$ \\
\hline $\begin{array}{l}\text { Growth } \\
\text { hormone } \\
(\mathrm{ng} / \mathrm{ml})\end{array}$ & $\begin{array}{r}0.13 \\
\pm 0.11\end{array}$ & $\begin{array}{r}0.18 \\
\pm 0.15\end{array}$ & $\begin{array}{l}1.52^{*} \dagger \\
\pm 1.17\end{array}$ & $\begin{array}{r}4.08^{*} \\
\pm 3.71\end{array}$ & $\begin{array}{r}0.45 \\
\pm 0.71\end{array}$ & $\begin{array}{r}0.33 \\
\pm 0.24\end{array}$ & $\begin{array}{r}0.28 \\
\pm 0.30\end{array}$ & $\begin{array}{l}3.27^{*} \\
\pm 2.83\end{array}$ \\
\hline $\begin{array}{l}\text { ANP } \\
\quad(\mathrm{pg} / \mathrm{ml})\end{array}$ & $\begin{array}{r}25.9 \\
\pm 9.8\end{array}$ & $\begin{array}{r}24.3 \\
\pm 7.9\end{array}$ & $\begin{array}{r}25.4 \\
\pm 7.4\end{array}$ & $\begin{array}{r}23.5 \\
\pm 7.2\end{array}$ & $\begin{array}{r}31.4 \\
\pm 5.6\end{array}$ & $\begin{array}{r}32.4 \\
\pm 3.5\end{array}$ & $\begin{array}{r}35.5 \\
\pm 8.1\end{array}$ & $\begin{array}{r}36.9 \\
\pm 7.8\end{array}$ \\
\hline $\begin{array}{r}\text { Kallikrein } \\
(\mathrm{ng} / \mathrm{ml})\end{array}$ & $\begin{array}{r}165.5 \\
\pm 67.7\end{array}$ & $\begin{array}{r}171.7 \\
\pm 99.9\end{array}$ & $\begin{array}{r}126.7 \\
\pm 76.3\end{array}$ & $\begin{array}{r}145.0 \\
\pm 91.9\end{array}$ & $\begin{array}{r}149.5 \\
\pm 42.1\end{array}$ & $\begin{array}{r}124.8 \\
\pm 23.8\end{array}$ & $\begin{array}{r}86.2 \\
+42.2\end{array}$ & $\begin{array}{r}118.3 \\
\pm 99.1\end{array}$ \\
\hline
\end{tabular}

Values are means \pm S.D..

${ }^{*} p<0.05$ vs. baseline values; ${ }^{* *} p<0.01$ vs. baseline values; $\dagger p<0.05$ vs. values after ingestion of egg white.

\section{Hormonal response to protein loading}

Plasma glucagon and growth hormone concentrations increased after ingestion of tuna fish or egg white (Table 5). Plasma concentrations of these two hormones increased significantly after ingestion of tuna fish. Plasma ANP was unchanged in response to both protein loadings. Urinary kallikrein excretion was also unchanged following both protein loads (Table 5).

\section{Discussion}

In the present study, GFR was evaluated on the basis of creatinine clearance, because evaluation of GFR by the radioisotope method is not legally recognized in Japan. Some investigators have already reported simultaneous inulin and creatinine clearance studies during a protein loading test and results obtained by both methods were comparable in patients without renal dysfuction (Bosch et al. 1983, 1984 ; Dhaene et al. 1987). Based on their findings, it seems likely that the results of GFR found in our study may be reliable.

The present study shows that ingestion of tuna fish caused a significant increase in GFR, whereas ingestion of the other three meals did not affect the GFR in healthy volunteers or diabetic patients. Therefore, the effects of protein administration on GFR may differ from one protein to another.

Intravenous administration of alanine, glycine and arginine increases GFR. In our study, plasma levels of these amino acids increased significantly only after ingestion of tuna fish, which caused an increase in the GFR. This result suggests that increase of GFR after ingestion of tuna fish may be due to increase of these 
amino acids in plasma. However, ingestion of the other meals did not induce increased plasma levels of these amino acids and glomerular hyperfiltration, despite the fact that these amino acids are as abundant in the other protein sources examined as in tuna fish. Furthermore, these amino acids were less contained in meat than tuna fish, though ingestion of meat induced hyperfiltration (Bosch et al. 1984 ; Hostetter et al. 1986). The mechanism behind these phenomenon remains to be elucidated. We cannot deny that these facts may due to differences in the intestinal absorption of meals.

Several mediators of the glomerular hyperfiltration observed after a protein meal have been reported. Glucagon, growth hormone, ANP and kallikrein induce glomerular hyperfiltration. In the present study, plasma glucagon and growth hormone concentrations increased significantly with increased GFR. However, plasma concentrations of these two hormones increased without glomerular hyperfitration, after administration of some protein. Furthermore, despite glomerular hyperfiltration, the plasma ANP concentration and urinary kallikrein excretion were unaffected by ingestion of two kinds of protein. These findings suggest that responses of GFR to acute protein loading may not be directly induced by these substances.

Recently, it was reported that dietary protein restriction prevented the progression of diabetic nephropathy. However, a low-protein diet may cause hypoproteinemia, muscle wasting, or malnutrition (Kleinknecht et al. 1979; Motomura et al. 1988). Moreover, it has been reported that not all proteins equally induce glomerular hyperfiltration (Dhaene et al. 1987). These facts led us to investigate whether diabetic nephropathy was influenced by the quality as well as the quantity of dietary protein. Our previous study showed that acute administration of vegetable proteins (at least of cooked soybean protein) did not increase GFR (Nakamura et al. 1989). In addition, the present study demonstrates that ingestion of cheese or egg white does not induce hyperfiltration. It seems probable, therefore, that these proteins can be exempted from dietary protein restriction. However, as our study involved only acute loading, prolonged clinical investigation is necessary to evaluate the possibility mentioned above. In prolonged diet therapy, the phosphorus content of cheese or bean curd should be taken into consideration, because phosphorus is unfavorable for renal function (Barsotti et al. 1984). Therefore, egg white should be used in clinical investigation of selective protein restriction.

\section{References}

1) Barsotti, G., Gambertoglio, J. \& Morelli, E. (1984) The decline of renal function slowed by very low phosphrus intake in chronic renal patients following a low protein diet. Clin. Nephrol., 21, 54-59.

2) Bosch, J.P., Saccaggi, A., Lauer, A., Ronco, C., Belledonne, M. \& Glabman, S. (1983) Renal function reserve in humans. Am. J. Med., 75, 943-950.

3) Bosch, J.P., Lauer, A. \& Glabman, S. (1984) Short-term protein loading in assess- 
ment of patients with renal disease. Am. J. Med., 77, 873-879.

4) Bosch, J.P., Lew, S., Glabman, S. \& Lauer, A. (1986) Renal hemodynamic changes in humans. Am. J. Med., 81, 809-815.

5) Corvilain, J. \& Abramow, M. (1962) Some effects of human growth hormone on renal hemodynamics and tubular phosphate transport in man. J. Clin. Invest., 41, 12301235 .

6) Dhaene, M., Sabot, J.P., Philippart, Y., Doutrelepont, J.M. \& Vanherweghem, J.L. (1987) Effect of acute protein loads from different sources on glomerular filtration rate. Kidney Int., 32, S25-S28.

7) Evanoff, G., Thompson, C., Brown, J. \& Weinman, E. (1987) The effect of dietary protein restriction on the progression of diabetic nephropathy. Arch. Intern. Med., 147, 492-495.

8) Fried, T.A. \& McCoy, R.N. (1986) Effects of atriopepsin II on determinants of glomerular filtration rate in the in vitro perfused dog glomerulus. Am. J. Physiol., 250, F1119-F1122.

9) Gingliano, D., Sicuranza, G., Quarto, E. \& Quartieri, J. (1986) Prevention of diabetic nephropathy by low-protein alimentation. In: Diabetic Renal Retinal Syndrome, Vol 3. edited by E.A. Friedman \& F.A. L’Esperance, Jr., Grune \& Stratton, Orlando, F.L., pp. 201-216.

10) Greene, S.A., Dalton, R.N., Turner, C., Haycock, G.B. \& Chantler, C. (1987) Hyperglycemia with and without glycosuria: Effect on inulin and para-amino hippurate clearance. Kidney Int., 32, 896-899.

11) Hostetter, T.H. (1986) Human renal response to a meat meal. Am. J. Med., 77,873879 .

12) Ito, S., Tsuda, A., Momotsu, T., Igarashi, K., Kasahara, S., Satoh, K. \& Shibata, A. (1989) Urinary orosomucoid excretion rate in patients with non-insulin-dependent diabetes mellitus. Acta Endocrinol., 120, 584-590.

13) Jones, G., Lee, K. \& Swaminathan, R. (1985) Glomerular filtration response to acute protein load. Lancet, 2, 838.

14) Kato, H., Adachi, N., Iwanaga, S., Abe, K., Takada, K., Kimura, T. \& Sakakibara, S. (1980) A new fluorogenic substrate method for the estimation of kallikrein in urine. J. Biochem., 87, 27-32.

15) Kleinknecht, C., Salusky, I. \& Broyer, M. (1979) Effects of various protein diets on growth, renal fuction and survival of uremic rats. Kidney Int., 15, 534-541.

16) Krishna, G.G., Newell, G., Miller, E., Hegger, P., Smith, R., Polansky, M., Kapoor, S. \& Hoeldtkt, R. (1988) Protein-induced glomerular hyperfiltration: Role of hormonal factors. Kidney Int., 33, 578-583.

17) Levy, S.B., Lilley, J.J., Frigon, R.P. \& Stone, R.A. (1977) Urinary kallikrein and plasma renin activity as determinants of renal blood flow. J. Clin. Invest., 60, 129136.

18) Mogensen, C.E. (1971) Glomerular filtration rate and renal plasma flow in short-term and long-term juvenile diabetes. Scand. J. Clin. Invest., 28, 91-100.

19) Mogensen, C.E. (1976) Renal fuction changes in diabetes. Diabetes, 25, 872-879.

20) Motomura K., Okida, S., Sanai, T., Ando, T., Onoyama, K. \& Fujishima, M. (1988) Importance of early initiation of dietary protein restriction for the prevention of experimental renal disease. Nephron, 49, 144-149.

21) Nakamura, H., Takasawa, M., Kasahara, S., Tsuda, A., Momotsu, T., Ito, S. \& Shibata, A. (1989) Effects of acute protein loads of different sources on renal function of patients with diabetic nephropathy. Tohoku J. Exp. Med., 159, 153-162.

22) Parving, H-H., Noer, J., Kehlet, H., Mogensen, C.E., Svensen, P.A. \& Heding, L. (1977) The effect of short term glucagon infusion on kidney function in normal man. Diabetologia, 13, 323-325.

23) Sugimoto, H. (1981) Fundamental and clinical studies on a new radioimmunoassay 
kit for the measurement of glucagon. Horm. Clin. Res., 29, 487-491. (Japanese)

24) Trevisan, R., Nosadini, R., Fioretto, P., Avogaro, A., Duner, E., Jori, E., Valerio, A., Doria, A. \& Crepaldi, G. (1987) Keton bodies increase glomerular filtration rate in anormal men and patients with type 1 (insulin-dependent) diabetes mellitus. Diabetologia, 30, 214-221.

25) Wiseman, M.J., Dodds, R., Bending, J.J. \& Viberti, G.C. (1987) Dietary protein and the diabetic kidney. Diabetic Med., 4, 144-146.

26) Yamaji, T., Ishibashi, M. \& Takaku, F. (1985) Atrial natriuretic factor in human blood. J. Clin. Invest., 76, 1705-1709. 